\title{
Thermodynamic parameterization
}

\author{
Alexander N. Gorban and Iliya V. Karlin \\ Nonequilibrium Systems Laboratory, Computing Centre, Russian Academy of Sciences, \\ Krasnoyarsk-36, 660036, Russian Federation
}

Received 27 August 1991

Revised manuscript received 28 April 1992

\begin{abstract}
A new method of successive construction of a solution is developed for problems of strongly nonequilibrium Boltzmann kinetics beyond normal solutions. Firstly, the method provides dynamic equations for any manifold of distributions where one looks for an approximate solution. Secondly, it gives a successive procedure of obtaining corrections to these approximations. The method requires neither small parameters, nor strong restrictions upon the initial approximation; it involves solutions of linear problems. It is concordant with the $H$-theorem at every step. In particular, for the Tamm-Mott-Smith approximation, dynamic equations are obtained, an expansion for the strong shock is introduced, and a linear equation for the first correction is found.
\end{abstract}

\section{Introduction}

In this paper we introduce a new approach to the problem of reduced description in dissipative kinetics. The method is addressed especially to those strongly non equilibrium problems which cannot be considered in frames of methods based on small parameters and normal solutions. In these problems we usually arrive at some special ad hoc approximations such as, for example, the well known Tamm-Mott-Smith (TMS) bimodal approximation. Even the most successful of these approximations make a dramatic impression due to that they are unbacked by any successive procedure (the problems of the TMS approximation are well known [1,2]).

We will consider the problem of reduced description for a general situation. Namely, we solve two main problems: (i) the problem of deriving a macroscopic dynamics for a given approximated reduced description, and (ii) the problem of obtaining corrections to this approximation. The resulting equations of the macroscopic description are thermodynamic (i.e. concordant with the $H$-theorem and conservation laws). The method of successive corrections 
avoids a consideration of small parameters, and it involves a Newton-type procedure based on linear equations.

Our method will be appropriate to any dissipative kinetic system with a global convex Lyapunov function (e.g. the Boltzmann equation provided with suitable boundary conditions, chemical kinetic equations for closed systems, the Fokker-Planck equation, etc). In order to make our consideration more certain, we will speak about the Boltzmann equation.

We suppose here that an approximated reduced description is chosen (it is unnecessary to discuss here particular reasons for this choice). This means that a manifold (a "surface") $\{f(a)\}$ is fixed in the space of distributions $f$, where $a$ represents coordinates on the manifold. For every $a$, the function $f(a)$ is the distribution which depends on spatial and momentum variables, and also on time. These latter dependencies will be omitted.

Here we arrive at two general problems:

(i) We must define macroscopic dynamics on the manifold $\{f(a)\}$. In order to do this, we must project (transform) the Boltzmann equation onto some macroscopic parameters. The first problem is: how and onto which macroscopic parameters should one perform this projection? In fact, there are a number of possible answers to this question. Which projector would make physical sense? We want that a solution of the first problem will preserve the thermodynamicity (i.e. the concordance with the $H$-theorem) at the chosen macroscopic level.

(ii) We understand that the chosen manifold is not a solution of the Boltzmann equation. In more general words, the chosen manifold $\{f(a)\}$ is not a dynamically invariant manifold of the Boltzmann equation. The notion "dynamically invariant manifold" appears in most of the dynamic theories: a manifold is called dynamically invariant if the vector field of the dynamical system is tangent to this manifold at every point.

Hence, we want to improve the chosen manifold $\{f(a)\}$ in order to make it "more invariant". The second problem is how to obtain these corrections in a general case (e.g. when there are no small parameters or other simplifications)? We hope that the solution of the second problem would be a method of successive approximations which would not require a too strong restriction upon the choice of the initial approximation $\{f(a)\}$.

Let us briefly outline this paper. In the next section we introduce a general construction which provides an essentially unique thermodynamic parameterization for the almost arbitrary manifold $\{f(a)\}$. In section 3 we introduce the invariance equation and we also introduce a Newton-type method to solve it. We obtain there an equation for the first correction to an arbitrary finite-dimensional approximation $\{f(a)\}$. In section 4 we clarify the physical sense of our approach. Lastly, in section 5, we consider the important 
particular case of the TMS approximation in the shock wave problem. There we obtain physically unique dynamic equations for this approximation, and we also obtain the equation for the first correction.

\section{Thermodynamic projector}

In this section we give a solution for the first of the two problems mentioned in the introduction (the problem of deriving the dynamic equations on the manifold $\{f(a)\}$ with preservation of thermodynamicity).

Our goal is to define macroscopic parameters $M$ as values of some operators $M(f)$ (yet unknown and, perhaps, nonlinear). The latter should be defined in a neighborhood of the manifold $\{f(a)\}$, and their values $M(f(a))=M(a)$ would create a new coordinate system on the manifold $\{f(a)\}$. We then obtain the expression of $\{f(a)\}$ as $\{f(M)\}$ where $M$ depends on $a$ (in the following we sometimes write $M$ instead of $M(a))$.

Knowing the operator $M(f)$, we are able to define dynamic equations on the manifold $\{f(a)\}$ :

$$
\mathrm{d} M(a) / \mathrm{d} t=\left.\int \nabla_{f} M(J(f))\right|_{f=f(M(a))} \mathrm{d}^{3} v .
$$

Here $\mathrm{d} / \mathrm{d} t$ represents the substantial derivative, and $J(f)$ is the Boltzmann collision integral.

As it was mentioned above, there are a number of a priori possibilities to choose the operator $M(f)$. However, any choice should satisfy the condition of thermodynamicity:

$$
\mathrm{d} H(M(a)) / \mathrm{d} t=\left(\left.\nabla_{M} H(M)\right|_{M=M(a)}, \mathrm{d} M(a) / \mathrm{d} t\right) \leqslant 0 .
$$

Here $H(M)=H(f(M))$, and $H(f)$ is the Boltzmann $H$-function. The notation $(.,$.$) is used for a scalar product in the space of macroscopic parameters M$. Condition (2) expresses that the Boltzmann $H$-theorem holds at the chosen macroscopic (reduced) level.

Hence, the basic point is to construct the operator (projector) $M(f)$ for a given manifold $\{f(a)\}$ which satisfies condition (2).

Our basic idea is to act as if a times hierarchy hypothesis (decomposition of motions) corresponds to the chosen approximation $\{f(a)\}$. This means that a "rapid" relaxation happens to the states $f(a)$ in a neighborhood of the manifold $\{f(a)\}$, and then "slow" motion along $\{f(a)\}$ takes place. The rapid motion determines the direction of projection. The choice of the projector is determined by the fact that the $H$-function decreases in rapid relaxation. In fact, 
the state $f$ from the neighborhood of $\{f(a)\}$ will be able to relax into the state $f(a)$ at the end of rapid motion if $f$ belongs to a hyperplane $\Gamma_{f(a)}$, the latter being orthogonal to the gradient of the $H$-function $\nabla_{f} H(f)$ at the point $f(a)$. We call $\Gamma_{f(a)}$ the hyperplane of rapid motion. For the $H$-function $\int f(\ln f-$ 1) $\mathrm{d}^{3} v$ we have $\nabla_{f} H(f)=\ln f$, and $\Gamma_{f(a)}$ is defined by the equation:

$$
\Gamma_{f(a)}=\left\{f \mid \int(f-f(a)) \ln f(a) \mathrm{d}^{3} v=0\right\} .
$$

We assume further that $\{f(a)\}$ is not tangent to a level of the $H$-function at any point $f(a) \in\{f(a)\}$. This is the only principal restriction on the choice of the manifold $\{f(a)\}$.

Thus, we define the functionals:

$$
M_{f(a)}^{*}(f)=\int f \ln f(a) \mathrm{d}^{3} v
$$

and the hyperplanes of rapid motions are defined as

$$
\Gamma_{f(a)}=\left\{f \mid M_{f(a)}^{*}(f-f(a))=0\right\} .
$$

It is clear that not all distributions of the hyperplane $\Gamma_{f(a)}$ are able to transform into the state $f(a)$ at the end of rapid motions. If the dimension of the manifold $\{f(a)\}$ is higher than one, then $\Gamma_{f(a)}$ includes some other distributions $f\left(a^{\prime}\right)$ for $a \neq a^{\prime}$. It is important that, for the validity of the macroscopic $H$-theorem (2), all rapid motions which lead to $f(a)$ should belong to $\Gamma_{f(a)}$. We will now show it.

Due to the strict convexity of the $H$-function, the point $f(a)$ is the only minimum of the $H$-function on the hyperplane of rapid motions $\Gamma_{f(a)}$. In other words, $f(a)$ coincides with the solution of the variational problem

$$
H(f) \rightarrow \min \quad \text { for } \int f \ln f(a) \mathrm{d}^{3} v=\int f(a) \ln f(a) \mathrm{d}^{3} v .
$$

Thus, the hypothesis of the times hierarchy for $\{f(a)\}$ means that the $H$-function decreases during the relaxation, and its minima occur on manifolds of rapid motions. The gradient of the $H$-function is normal to this manifold of rapid motions at the minimum. Therefore, in the linear approximation the equation

$$
\int f \ln f(a) \mathrm{d}^{3} v=\int f(a) \ln f(a) \mathrm{d}^{3} v
$$

is valid for those distributions $f$ which are able to relax to the state $f(a)$ in rapid processes. 
We finish the construction of the projector by choosing macroscopic parameters $M$ which define a coordinate system on the manifold $(f(a)\}$. As a rule, it is sufficient to add a system of linear functionals $L(f)=\int l(v) f \mathrm{~d}^{3} v$ to the functionals $M_{f(a)}^{*}(f)$ (4) (further we consider only this case; however, the linearity does not play any specific role). Then the manifold $\{f(a)\}$ will be parameterized by the macroscopic parameters $M_{f(a)}^{*}(f(a))$ and $L(f(a))$. Any choice of macroscopic parameters additional to $M_{f(a)}^{*}(f(a))$ does not disturb the solution of the variational problem (6). Indeed, additional functionals $L(f)$ give only additional restrictions for the problem (6). These conditions, $L(f-$ $f(a))=0$, "cut out" manifolds of lower dimension inside the hyperplane of rapid motions (3). One can consider the resulting linear manifold as a linear manifold of rapid motions which lead exactly to the distribution $f(a)$. Hence, we see that the distribution $f(a)$ is also the unique solution of the problem

$$
H(f) \rightarrow \min \quad \text { for } M_{f(a)}^{*}(f-f(a))=0, \quad L(f-f(a))=0 .
$$

Thus, for the manifold $\{f(a)\}$, we have defined the operator $M(f)$ as the set of functionals $\left(M_{f(a)}^{*}(f), L(f)\right)$. Substituting these latter into (1) yields the resulting set of dynamic equations.

The functionals $-k_{\mathrm{B}} M_{f(a)}^{*}$ project the points $f(a)$ into the values of the entropy $S(f(a))$ and determine the entropy balance equation on the manifold $\{f(a)\}$. Thus, the times hierarchy hypothesis results immediately in the thermodynamic parameterization of the manifold $\{f(a)\}$.

An important particular case occurs when the manifold considered is a quasi-equilibrium manifold (i.e. $f(M)$ is the solution of the problem $H(f) \rightarrow \min$ for $M(f)=M$, where $M(f)$ is fixed prior to obtaining $f(M)$ ). Here one does not need a new projector. The quasi-equilibrium manifold $\{f(M)\}$ is thermodynamic due to its construction [3-5]: $\ln (f(M))$ then is a linear combination of $\left.\nabla_{f} M(f)\right|_{f=f(M)}$. Due to (6) one can consider an arbitrary manifold as if it were a quasi-equilibrium manifold after the appropriate parameterization. It should be stressed that in (6) we solve a reversed problem: we start with the manifold $\{f(a)\}$ and next we construct the operator $M(f)$.

In spite of the external simplicity of the final results (the entropy balance equation is indeed "natural"), this parameterization has a complicated structure because the functionals $M_{f(a)}^{*}(f)(4)$ are neither the usual moment functional nor the entropy.

We use the asterisks ${ }^{*}$ in order to stress the thermodynamicity of parameterization.

Thus, for a given manifold $\{f(a)\}$ we have constructed the thermodynamic parameterization $\left\{f^{*}(M(a))\right\}$ concordant with the condition (2). The functionals (4) play the key role. In the next section we consider the problem of constructing a dynamically invariant manifold from the manifold $\{f(a)\}$. 


\section{Dynamic invariance}

We start with a formalization of the requirement on dynamic invariance for a manifold $\{f(M)\}$ (it is important that the manifold is parameterized by macroscopic parameters, cf. in section 2).

We write the Boltzmann equation as

$$
\frac{\partial f(\boldsymbol{v}, \boldsymbol{x}, t)}{\partial t}=B(f), \quad B(f)=-\boldsymbol{v} \frac{\partial f(\boldsymbol{v}, \boldsymbol{x}, t)}{\partial \boldsymbol{x}}+J(f) .
$$

Denote by $S_{\mathrm{t}}$ the operator of transition along a solution of the Boltzmann equation for the time $t \geqslant 0$. A manifold $\{f(M)\}$ is called dynamically invariant with respect to the Boltzmann equation if for all $M$ and $t \geqslant 0$ the following equality is valid:

$$
S_{\mathrm{t}} f(M)=f\left(M\left(S_{\mathrm{t}} f(M)\right)\right) .
$$

The equality (9) has a clear geometrical interpretation: the Boltzmann dynamics translates the point $f(M)$ into another point $f\left(M^{\prime}\right)$ on the same manifold.

The differential version of the invariance equation (9) is more useful:

$$
\Delta(f(M))=\left(\nabla_{M} f(M), \partial M(f(M)) / \partial t\right)-B(f(M))=0 .
$$

Here $\partial M(f(M)) / \partial t$ represents the derivative of the macroscopic parameters caused by eq. (8) at the point $f(M)$ (i.e. the partial time derivative is expressed through the macroscopic parameters $M$ from the macroscopic dynamical equations of type (1)).

We consider the condition of dynamic invariance (10) as a nonlinear equation which one should solve by a method of successive approximations. The method should preserve thermodynamicity at every iteration.

We introduce a Newton-type method of successive approximations to solve the invariance equation (10). This procedure involves a linearization of the vector field $B(f)$, and no small parameters are required for this linearization.

The complete scheme of the method of obtaining corrections to the initial manifold $\left\{f_{0}(a)\right\}$ involves the following steps:

Step 1. Choose the initial approximation $\left\{f_{0}(a)\right\}$.

Step 2. Create a thermodynamic parameterization $\left\{f_{0}^{*}(M)\right\}$ as described in section 2.

Step 3. Calculate the defect $\Delta\left(f_{0}^{*}(M)\right)$. If $\Delta\left(f_{0}^{*}(M)\right)$ is identical to zero, then we have the dynamic invariant manifold $\left\{f_{0}(a)\right\}$. If not, then linearize the 
operator $B(f)$ in $(10)$ in the neighborhood of the manifold $\left\{f_{0}(a)\right\}$, and solve the linear equation obtained (remember that operator $B(f)$ appears also in the expression $\partial M(f(M)) / \partial t)$. The solution is a new manifold $\left\{f_{1}(a)\right\}$.

Step 4. Create the thermodynamic parameterization $\left\{f_{1}^{*}(M)\right\}$ as described in section 2 .

Then the process is continued (go to step 3).

We will finish this section by considering in more detail the important particular case of finite-dimensional manifold $\{f(a)\}$.

For an $r$-parametric manifold $\{f(a)\}$, where $a=\left(a_{1}, \ldots, a_{r}\right)$, we consider the case of thermodynamic parameterization when the functionals $M_{f(a)}^{*}(f)(4)$ are completed with $r-1$ independent linear functionals:

$$
L_{i}(f)=\int l_{i}(v) f \mathrm{~d}^{3} v . \quad i=1, \ldots, r-1 .
$$

Equations of reduced description (1) are as follows:

$$
\begin{aligned}
& \frac{\partial H(a)}{\partial t}+\operatorname{div} j_{H}(a)=\sigma(a), \quad \frac{\partial L_{i}(a)}{\partial t}+\operatorname{div} j_{L_{i}}(a)=R_{i}(a) \\
& H(a)=\int f(a)[\ln f(a)-1] \mathrm{d}^{3} v, \quad L_{i}(a)=\int l_{i}(\boldsymbol{v}) f(a) \mathrm{d}^{3} v, \\
& j_{H}(a)=\int \boldsymbol{v} f(a)[\ln f(a)-1] \mathrm{d}^{3} v, \quad j_{L_{i}}(a)=\int v l_{i}(v) f(a) \mathrm{d}^{3} v, \\
& \sigma(a)=\int \ln f(a) J(f(a)) \mathrm{d}^{3} v, \quad R_{i}(a)=\int l_{i}(v) J(f(a)) \mathrm{d}^{3} v .
\end{aligned}
$$

At the first iteration we search for

$$
\ln f_{1}^{*}=\ln f_{0}^{*}+\varphi_{1} .
$$

The correction $\varphi_{1}$ is obtained in order that the new manifold $f_{1}^{*}=f_{0}^{*} \exp \left(\varphi_{1}\right)$ will satisfy the invariance equation (10) within an accuracy of $\varphi_{1}$ order terms. Thus, $\varphi_{1}$ is obtained from the following linear in $\varphi_{1}$ equation:

$$
\left(1-P_{0}\right) B_{\operatorname{lin}}\left(f_{0}^{*}(M) ; \delta f_{1}\right)=\Delta\left(f_{0}^{*}(M)\right) .
$$

Here $B_{\text {lin }}\left(f_{0}^{*}(M) ; \delta f_{1}\right)$ represents the linearization of the Boltzmann vector field at the point $f_{0}^{*}(M)$, and $\delta f_{1}=f_{0}^{*}(M) \varphi_{1}$. The operator $P_{0}$ acts as follows:

$$
P_{0} g=\frac{\partial f_{0}^{*}}{\partial H_{0}} \int g \ln f_{0} \mathrm{~d}^{3} v+\sum_{i=1}^{r-1} \frac{\partial f_{0}^{*}}{\partial L_{i 0}} \int l_{i}(v) g \mathrm{~d}^{3} v
$$


The notation $\partial f^{*} / \partial M$ represents the derivative

$$
\left.\frac{\partial f(a(M(f))}{\partial M(f)}\right|_{M(f)=M(f(a))} \text {. }
$$

The equation of the $k$ th iteration is obtained from eqs. (13) and (14) by the rearrangement of indices $0 \rightarrow k-1,1 \rightarrow k$. Immediate calculations show that $P_{k}^{2}=P_{k}$, i.e. that the operators $P_{k}$ are projecting operators. They project onto the tangential space of the manifold of the $k$ th approximation.

\section{Physical interpretation}

We are now going to discuss the intuitive ideas behind our approach. The method is based on two points: (i) thermodynamic parameterization, and (ii) a successive correction of the dynamic noninvariance. These points are the immediate formalization of two general principles of dissipative kinetics: (i) the choice of an approximated reduced description always involves an implicit assumption on the decomposition of times of relaxation, and (ii) a dynamic invariant manifold of slow motions is located in a neighborhood of the chosen approximation. This is rather a fine place, and it requires additional explanations.

Usually when one talks about decomposition of motions (i.e. about the times hierarchy), one keeps in mind the existence of a small parameter. This small parameter should express the ratio of the time of rapid relaxation to the time of macroscopic observation. One may expect that the result of the rapid relaxation will be a "sufficiently good" manifold of slow motions (i.e. it will be a "sufficiently invariant" manifold).

However, this situation is far from simple. There is always doubt on whether the chosen parameter is sufficiently small. Even for finite-dimensional dissipative systems (e.g. chemical kinetics) the steady-state manifolds might not always be referred as to good approximations (see a precise study: "The steady-state approximations, fact or fiction?" by E. Farrow and D. Edelson [6] and also ref. [7]).

On the other hand, there are no small parameters in the general case, but still one can construct a "good" approximation which approximately describes the evolution for a considerable period. For example, the TMS approximation illustrates this situation: a small parameter lacks in the strong shock wave problem but, nevertheless, one can consider the TMS approximation as a suitable approximation for this problem. Hence, we should take that the two assumptions mentioned above are appropriate to the TMS approximation (at least approximately). 
The problem of dynamic invariant manifolds has a very specific sound for dissipative kinetics. Namely, one should expect that these manifolds are manifolds of slow motion.

For a chosen approximated reduced description, one can not say beforehand whether the decomposition of motions indeed corresponds to the choice. Nevertheless, we act as if the chosen manifold is already a "good" manifold of slow motions. This immediately leads to the definition of hyperplanes of rapid motions via the principles of decrease of the $H$-function in rapid relaxation as described above. It is important that the variational principle (6) avoids a search for small parameters for the conduction of the manifolds of rapid motions. We obtain thermodynamic parameterization for the initial manifold. At the same time we remember that the chosen approximation is not a dynamic invariant manifold. We are able to measure the error caused by noninvariance from the definition (10), and we are able to approximately correct this error by solving the linear equation of the first Newton iteration (13). Then we again act as if the corrected manifold is a good manifold of slow motions, etc.

It is very important that no small parameters are required to complete the procedure. The process is the same in its character for any initial approximation. Small parameters can be used for simplification in some cases.

\section{Example: the Tamm-Mott-Smith approximation}

The following (I. Tamm and H. Mott-Smith) approximation is commonly known in the shock wave problem [1]:

$$
f\left(a_{-}, a_{+}\right)=a_{-} f_{-}+a_{+} f_{+} .
$$

Here $f_{-}$and $f_{+}$represent Maxwell distributions infinitely far up and down the flow. According to refs. [1,2], the choice of the "natural" projector is the main unsolved problem of the approximation (15).

Thermodynamic parameterization of the approximation (15) is determined by the values of the functionals $M_{f\left(a_{-}, a_{+}\right)}^{*}(f)(4)$ and of the moment functional $n(f)=\int f \mathrm{~d}^{3} v$. Then the dynamics of $a_{-}$and $a_{+}$are obtained from the equations for the macroscopic variables $M_{f\left(a_{-}, a_{+}\right)}^{*}\left(f\left(a_{-}, a_{+}\right)\right)$and $n\left(f\left(a_{-}, a_{+}\right)\right)$ (see (12)):

$$
\frac{\partial s}{\partial t}+\frac{\partial j_{s}}{\partial x}=\sigma, \quad \frac{\partial n}{\partial t}+\frac{\partial j_{n}}{\partial x}=0, \quad s=-k_{\mathrm{B}} \int f_{0}^{*} \ln f_{0}^{*} \mathrm{~d}^{3} v
$$




$$
\begin{aligned}
& n=\int f_{0}^{*} \mathrm{~d}^{3} v, \quad j_{s}=-k_{\mathrm{B}} \int v_{x} f_{0}^{*} \ln f_{0}^{*} \mathrm{~d}^{3} v, \quad j_{n}=\int v_{x} f_{0}^{*} \mathrm{~d}^{3} v, \\
& \sigma=-k_{\mathrm{B}} \int \ln f_{0}^{*} J\left(f_{0}^{*}\right) \mathrm{d}^{3} v .
\end{aligned}
$$

Here $f_{0}^{*}$ stands for $f\left(a_{-}, a_{+}\right)$.

The stationary version of the set (16) was originally introduced ad hoc by $M$. Lampis in ref. [8]. Here we have shown that eqs. (16) are the only equations which make physical sense in the Tamm-Mott-Smith kinetics.

A detailed investigation of eqs. (16) is not the goal of this paper. Here we will indicate an approach to the analysis of the strong stationary shock wave (the more simple case of the weak wave was considered in ref. [8]). We assume that the wave propagates along the $x$-axis. We divide the velocity space into two regions: $V_{-}\left(a_{-} f_{-}>a_{+} f_{+}\right)$and $V_{+}\left(a_{-} f_{-}<a_{+} f_{+}\right)$. Then we introduce the following expansion:

$$
\ln \left(a_{-} f_{-}+a_{+} f_{+}\right)= \begin{cases}\ln \left(a_{-} f_{-}\right)+a_{+} f_{+} / a_{-} f_{-}-\cdots & \text { for } a_{-} f_{-}>a_{+} f_{+} \\ \ln \left(a_{+} f_{+}\right)+a_{-} f_{-} / a_{+} f_{+}-\cdots & \text { for } a_{-} f_{-}<a_{+} f_{+}\end{cases}
$$

The surface which separates $V_{-}$from $V_{+}$is obtained from the condition of term-by-term continuity of the expansion (17). This surface does not depend on the number of terms taken into account. The equation of this surface is

$$
\begin{aligned}
& \sum_{i=y, z} v_{i}^{2}+\left(v_{X}-\frac{u_{-} T_{+}-u_{+} T_{-}}{T_{+}-T_{-}}\right)^{2}=r^{2}+\rho, \\
& \rho=\frac{2 R T_{-} T_{+}}{M\left(T_{+}-T_{-}\right)} \ln \left(\frac{a_{-} u_{+}}{\left(1-a_{-}\right) u_{-}}\right), \\
& r^{2}=\frac{T_{-} T_{+}\left(u_{+}-u_{-}\right)^{2}}{\left(T_{+}-T_{-}\right)^{2}}+\frac{3 R T_{-} T_{+}}{M\left(T_{+}-T_{-}\right)} \ln \left(\frac{T_{+}}{T_{-}}\right) .
\end{aligned}
$$

Here $R$ is the gas constant, $M$ is the molar mass, $u_{\mp}$ and $T_{\mp}$ represent the hydrodynamic quantities infinitely far up and down the flow. The expansion (17) solves the main difficulty of analytic investigation of eqs. (16). This difficulty appears from the logarithm of the sum of functions under integration.

Finally, we introduce the equation of the first iteration (13) for the TMS initial approximation. The operator $P_{0}(14)$ acts as follows:

$$
P_{0} g=\frac{\partial f_{0}^{*}}{\partial H_{0}} \int g f_{0} \mathrm{~d}^{3} v+\frac{\partial f_{0}^{*}}{\partial n_{0}} \int g \mathrm{~d}^{3} v, \quad \frac{\partial f_{0}^{*}}{\partial H_{0}}=Z\left(\varphi_{+}-\varphi_{-}\right),
$$




$$
\frac{\partial f_{0}^{*}}{\partial n_{0}}=Z\left(\varphi_{+}\left\langle\ln f_{0}\right\rangle_{-}-\varphi_{-}\left\langle\ln f_{0}\right\rangle_{+}\right), \quad Z=\left(\left\langle\ln f_{0}\right\rangle_{+}-\left\langle\ln f_{0}\right\rangle_{-}\right)^{-1}
$$

Notations used are: $\varphi_{ \pm}=f_{ \pm} / n_{ \pm},\langle g\rangle_{ \pm}=\int g \varphi_{ \pm} \mathrm{d}^{3} v$. The defect of the approximation (17) (the RHS of eq. (13)) is equal to

$$
\begin{aligned}
\Delta_{0}= & Z\left[\varphi_{-}\left(-\left\langle\ln f_{0}\right\rangle_{+} \frac{\partial j_{n_{0}}}{\partial x_{1}}+\frac{\partial j_{H_{0}}}{\partial x_{1}}-\sigma_{0}\right)\right. \\
& \left.-\varphi_{+}\left(-\left\langle\ln f_{0}\right\rangle_{-} \frac{\partial j_{n_{0}}}{\partial x_{1}}+\frac{\partial j_{H_{0}}}{\partial x_{1}}-\sigma_{0}\right)\right]+v_{1} \frac{\partial f_{0}}{\partial x_{1}}-J\left(f_{0}\right) .
\end{aligned}
$$

Thus, eq. (13), with the account of (19) and (20), gives the linear equation for the first correction to the Tamm-Mott-Smith approximation.

\section{Conclusions and remarks}

(i) The method of constructing the projector introduced in section 2 solves the problem of thermodynamic parameterization. The condition of the entropy growth during rapid relaxation results in the entropy balance equation for the slow process. One can prove that there is no other universal way to construct a thermodynamic parameterization of an arbitrary manifold. In particular, eqs. (16) solve the old problem of ambiguousness of the Mott-Smith approximation.

(ii) When constructing the projector we have neglected the deviations from $\{f(a)\}$ in the slow process. As was mentioned above, we act as if the manifold $\{f(a)\}$ is the manifold of slow motion. Accurately speaking, this is not so. The next step should be the obtainment of corrections for the approximation $\{f(a)\}$. The construction of the thermodynamic projector is the preparation of the general Newton-type procedure of constructing a dynamic invariant manifold from a given initial approximation introduced in section 3 .

\section{Acknowledgements}

The authors wish to thank Prof. C. Cercignani for his attention to this work and for informing us about paper [8]. We are also grateful to the referee of "Physica A" for helpful remarks. This work was supported by the USSR-USA Foundation "Cultural Initiative" (the grant "Provincial Science of Siberia"). 


\section{References}

[1] C. Cercignani, Theory and Applications of the Boltzmann Equation (Scottish Academic Press, Edinburgh, 1975).

[2] R. Caflish, in: Nonequilibrium Phenomena I (North-Holland, Amsterdam, 1983) pp. $192-223$.

[3] A.M. Kogan and L.I. Rozonoer, Sov. Math. Rev. 158 (1964) 566.

[4] A.N. Gorban, Equilibrium Encircling (Nauka, Novosibirsk, 1984).

[5] I.V. Karlin, in: Mathematical Problems of Chemical Kinetics (Nauka, Novosibirsk, 1989) pp. $7-42$.

[6] E. Farrow and D. Edelson, Int. J. Chem. Kinet. 6 (1974) 787.

[7] G.S. Yablonskii, V.I. Bykov, A.N. Gorban and V.I. Elokhin, Kinetic Models of Catalytic Reactions, Comprehensive Chemical Kinetics, vol. 32 (Elsevier, Amsterdam, 1991).

[8] M. Lampis, Meccanica 12 (1977) 171. 